\section{In-frame deletion of Escherichia coli essential genes in complex regulon}

\author{
Rubing Liang and Jianhua Liu \\ College of Life Science and Technology, Shanghai Jiaotong University, Shanghai, China
}

BioTechniques 44:209-215 (February 2008)

doi 10.2144/000112687

\begin{abstract}
A conditional knockout-rescue system was developed to construct an in-frame deletion strain of Escherichia coli essential genes. The target was flanked with marker genes and FRT (FLP recognition target) sites, and a plasmid containing arabinose-induced FLP recombinase was transformed. After arabinose induction, cells could survive only when target protein activity was provided in trans. We selected three essential genes as targets, yaeT, fabZ, and dnaE, which are components of the complex eight-gene regulon yaeT-hlpA-lpxD-fabZ-lpxA-lpxBrnhB-dnaE. Deletion of these three genes exhibit no polar effects on their adjacent genes in terms of cell viability, meaning that this system not only allows for the simplified study of protein interactions and homolog screening in other organisms, but also facilitates the null mutant construction of essential genes.
\end{abstract}

\section{INTRODUCTION}

Because of their core functions and evolutionary conservation, essential genes are of particular interest and serve as starting points for minimal genome designs (1). However, the difficulties in essential gene mutation have impeded their study. Many approaches have been developed to study gene function, such as temperature-sensitive (TS) mutants $(2,3,4)$, controlled gene expression $(5,6)$, RNA interference (7), and tRNA suppression (8). However, leaky gene expression (9), design difficulties $(2,3,4)$, laborious work (8), and unregulated expression $(5,6)$ have hindered their application.

The eight-gene regulon yaeT-hlpAlpxD-fabZ-lpxA-lpxB-rnhB-dnaE at $4.30 \mathrm{~min}$ on the Escherichia coli genome, is complex, containing six essential genes that participate in DNA replication, repair, immune reaction, and signal transduction. The $f a b Z$ gene encodes a (3R)-hydroxymyristol acyl carrier protein dehydratase, and the lpxD-fabZ-lpxA gene cluster is involved in lipid A biosynthesis in many bacteria $(10,11)$. YaeT, a highly conserved protein (12), is required for gram-negative outer membrane protein assembly $(13,14,15,16)$. In cells lacking YaeT, insertion, folding, and oligomerization of outer membrane $d n a E$ gene encodes the alpha-catalytic subunit of the DNA polymerase III holoenzyme. The TS dnaE allele blocks DNA synthesis at the initiation stage of DNA replication and elongation proteins are defective $(17,18)$. The
$(19,20)$. Although many studies about this operon have been reported, null mutants have not been obtained.

In this work, a conditional knockoutrescue system for the in-frame deletion of essential genes was established. Using this system, three deletion strains of yaeT, fabZ, or dnaE genes were constructed and the polarity effects of deletion were investigated.

\section{MATERIALS AND METHODS}

\section{Strains, Plasmids, Media, and Culture Conditions}

Strains and plasmids are listed in Table 1. M63 minimal medium (21) contained 18 amino acids (no methionine or cysteine), thiamine $(1 \mu \mathrm{g} / \mathrm{ml})$, and $0.2 \%$ of a specified carbon source (22). For agar plates, $10 \mathrm{~g}$ agar were added per liter and both $100 \mu \mathrm{g} / \mathrm{ml}$ ampicillin and $10 \mu \mathrm{g} / \mathrm{ml}$ tetracycline were used for antibiotic resistance selection. Sucrose was added at a concentration of $5 \%$. E. coli DH5 $\alpha$
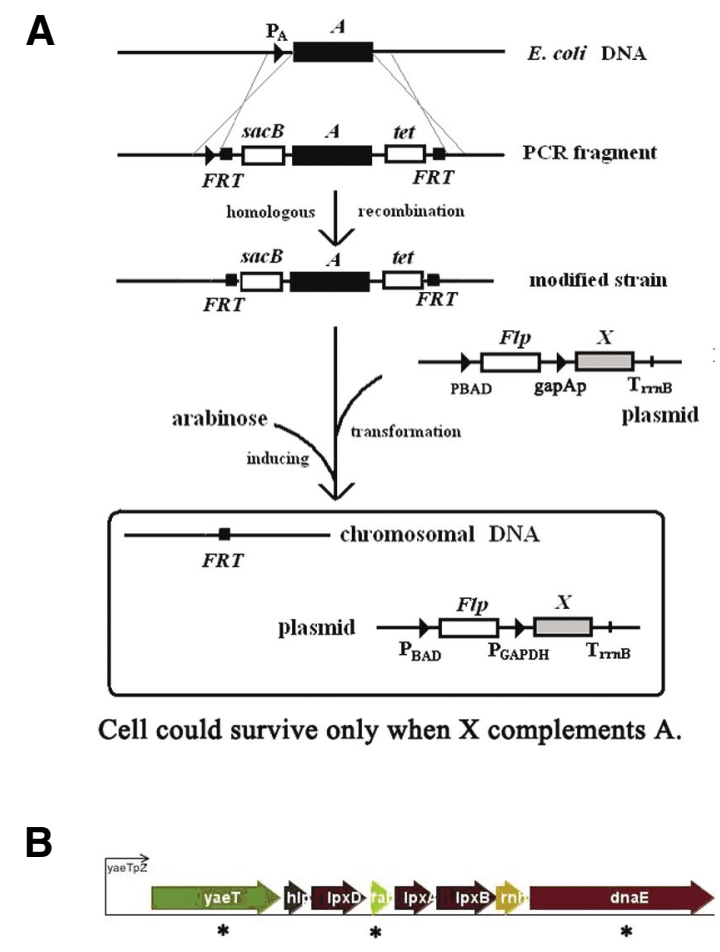

Figure 1. System strategy and structure of eight-gene operon. (A) Strategy. A, chromosomal target essential gene; tet, positive marker gene; $s a c B$, negative marker gene; $X$, sample gene. (B) The yaeT-hlpAlpxD-fabZ-lpxA-lpxB-rnhB-dnaE operon structure and the three target genes marked with an asterisk. 
Table 1. Strains and Plasmids

\begin{tabular}{|c|c|c|}
\hline Name & Description & Source/Reference \\
\hline \multicolumn{3}{|l|}{ Strains } \\
\hline DY329 & $\begin{array}{l}\text { Lam-, IN (rrnD-rrnE) 1, rph-1 } \Delta \text { "'acU169 nadA::Tn10 } \\
\text { gal490 } \lambda c / 857 \Delta \text { (cro-bioA) }\end{array}$ & $(25)$ \\
\hline $\mathrm{DH} 5 \alpha$ & $\begin{array}{l}\mathrm{F}^{\prime} / \text { endA1 hsdR17 }\left(\mathrm{r}_{\mathrm{K}}-\mathrm{m}_{\mathrm{K}}{ }^{-}\right) \text {glnV44 thi-1 recA1 gyrA } \\
(\text { NalR) relA1 } \Delta(\text { (laclZYA-argF)U169 deoR (Ф80dlacs } \\
\text { (lacZ)M15) }\end{array}$ & (23) \\
\hline DY1 & DY329, $\Delta$ yecl:: (PBAD-Flp-sacB-bla), Sucs, Apr & This work \\
\hline DY2 & $\mathrm{DY} 1, \Delta(\mathrm{sacB}-b / a), \mathrm{Suc}^{\mathrm{r}}, \mathrm{Ap}^{\mathrm{s}}$ & This work \\
\hline DYSB & DY2, (FRT-sacB-tet-FRT), Sucs, Tcr & This work \\
\hline DYAY & DY329, $\Delta$ yecl:: (FRT-sacB-tet-FRT), Sucs, $\mathrm{Tc}^{r}$ & This work \\
\hline FSYTF & DY329, $\Delta y a e T::(F R T$-sacB-yaeT-tet-FRT), Sucs, Tcr & This work \\
\hline FSFTF & DY329, $\Delta f a b Z::(F R T$-sacB-fabZ-tet-FRT), Sucs, Tcr & This work \\
\hline FSDTF & DY329, $\Delta d n a E::\left(F R T\right.$-sacB-dnaE-tet-FRT), Suc ${ }^{s}, \mathrm{Tc}^{r}$ & This work \\
\hline \multicolumn{3}{|l|}{ Plasmids } \\
\hline pEX18Tc & $\begin{array}{l}\text { oriT+, sacB+, gene replacement vector with MCS from } \\
\text { pUC18, Sucs, Tcr }\end{array}$ & $(24)$ \\
\hline pPS854 & $\begin{array}{l}\text { Vector containing two FRT sites and MCS from } \\
\text { pUC18, Ap }\end{array}$ & $(24)$ \\
\hline pFLP2 & $\begin{array}{l}\text { Vector containing cl857-controlled FLP encoding } \\
\text { sequence, Sucs, Apr }\end{array}$ & $(24)$ \\
\hline pBAD18 & Vector containing PBAD operon, $\mathrm{Ap}^{r}$ & $(5)$ \\
\hline pPST & $\begin{array}{l}\text { pPS854 with sacB and tet inserted into } F R T \text { sites, } \\
\text { Tcr }, A p^{r}\end{array}$ & This work \\
\hline pPST1 & pPST, yaeT & This work \\
\hline pPST2 & pPST, fabZ & This work \\
\hline pPST3 & pPST, dnaE & This work \\
\hline pBFLP & pBAD18, Flp & This work \\
\hline pBFLPE & pBFLP, gapAp & This work \\
\hline pBFLPE1 & pBFLPE, yaeT & This work \\
\hline pBFLPE2 & pBFLPE, yae $T, h / p A$ and $I p x D$ & This work \\
\hline pBFLPE3 & pBFLPE, fabZ & This work \\
\hline pBFLPE4 & pBFLPE, IpxD and $f a b Z$ & This work \\
\hline pBFLPE5 & pBFLPE, fabZ and $I p x A B$ & This work \\
\hline pBFLPE6 & pBFLPE, dnaE & This work \\
\hline pBFLPE7 & pBFLPE, $r n h B$ and $d n a E$ & This work \\
\hline
\end{tabular}

cells were grown at $37^{\circ} \mathrm{C}$ and strains derived from DY329 cells were grown at $32^{\circ} \mathrm{C}$.

\section{PCR and Oligonucleotides}

PCR (polymerase chain reaction) was performed with LA Taq DNA polymerase according to the manufacturer's protocol (TaKaRa Biocompany, Dalian, China). DNA sequences of the PCR primers are listed in Table 2. Lyophilized primers were synthesized by Invitrogen Ltd. (Shanghai, China), dissolved in $\mathrm{H}_{2} \mathrm{O}$, and stored at $-20^{\circ} \mathrm{C}$. General techniques for plasmid DNA preparation, restriction enzyme manip- ulation, molecular cloning, and agarose gel electrophoresis were carried out per standard protocols. Restriction endonuTaKaRa Biocompany. Plasmid transformations and preparations were done in E. coli DH5 $\alpha$ cells (23).

\section{Plasmid Construction}

The Flp-sacB fragment amplified from pFLP2 (24) was inserted into the EcoRI/HindIII site of pBAD18 the arabinose-controlled $F l p$ and $s a c B$ genes. After BamHI/HindIII digestion and ligation, the gapAp fragment cleases and ligase were purchased from (5) to construct pBFLP, containing replaced the $s a c B$ gene of $\mathrm{pBFLP}$ to produce pBFLPE containing the gapA promoter, $R B S$ (ribisome binding sequence), and several restriction sites (SphI, SacI, SalI, KpnI, XhoI). Plasmid pBFLPE1-7 was generated by ligation of XhoI/SphI-digested pBFLPE with different cassettes, yaeT, yaeT-hlpAlpxD, fabZ, lpxD-fabZ, fabZ-lpxAB, dnaE, and $r n h B-d n a E$, which could express these gene(s) constitutively.

pPST was generated by inserting the EcoRI/EcoRV-digested sacB-tet fragment from pEX18Tc (24) into pPS854, which had been treated identically (24). pPST1-3 containing a $F R T$-sacB-target-tet-FRT cassette was constructed by the ligation of SspItreated yaeT, fabZ, and dnaE fragments into the pre-digested pPST.

\section{Strain Construction}

Strains were constructed by homologous recombination as described previously (25). DY1 was generated by replacing the yecI gene of DY329 with the PBAD-Flp-sacB-bla cassette amplified from pBFLP. DY2 was engineered from DY1 by deleting the marker genes and DY2 was modified to produce DYSB by inserting the FRT-sacB-tet-FRT (sb) cassette into the downstream Flp gene. DYAY was generated by replacing the yecI gene of DY329 with the FRT-sacB-tet-FRT (ay) cassette. FSYTF, FSFTF, and FSDTF were constructed separately by substituting the yaeT, fabZ, and dnaE genes of DY329 for the FRT-sacB-fabZ-tetFRT (FSYTF), FRT-sacB-fabZ-tet-FRT (FSFTF), and FRT-sacB-dnaE-tet-FRT $(F S D T F)$ fragments.

\section{System Efficiency Detection}

pPST and pBFLPE were transformed into DY2 and DYAY, respectively. The two transformants and DYSB were inoculated into $1 \mathrm{ml}$ of M63 medium and cultured at $32^{\circ} \mathrm{C}$ until the $\mathrm{OD}_{600}$ reached approximately 0.3 . Cells were harvested and spread on M63 plates containing ampicillin and arabinose of different concentrations (from $0.05 \%$ to $0.5 \%$ ). Control experiments were performed with glycerol as the inducer. The following day, 100 colonies were picked from each plate and screened in 


\section{Short Technical Reports}

Table 2. Sequences of PCR Primers

\begin{tabular}{|c|c|c|}
\hline Name & Sequence & Description \\
\hline 1 & AAGGTCGAATTCactattttacctctggcggt & Amplification of $F / p$-sacB cassette \\
\hline 3 & GGGGGGGGATCCgctcacatctcactttaatcg & \multirow[t]{2}{*}{ Amplification of gapAp fragment } \\
\hline 4 & GGGGGAAGCTTGCATGCGAGCTCGTCGACGGTACCTCGAGCCACCAgctatttgttagtg & \\
\hline 5 & AAGGTCGAATTCggagacatgaacgatgaacatc & \multirow[t]{2}{*}{ Amplification of sacB-tet cassette } \\
\hline 6 & TTGCATGATATCtccattcaggtcgaggtgg & \\
\hline 10 & cgctgaatgccgatcaggaa & $\begin{array}{l}\text { Amplification of } s a c B \text {-bla deletion } \\
\text { cassette }\end{array}$ \\
\hline 11 & GTACTAGACTACCTTTCATCCTACATAAATAGACGCATATgagctcgaattggggatctt & \multirow{2}{*}{$\begin{array}{l}\text { Amplification of } F R T \text {-sacB-tet-FRT } \\
\text { (sb) cassette }\end{array}$} \\
\hline 12 & GTCCGAAATGTGATCGTGGAGTCAATTCTGACGATGATgaattagcttcaaaagcgctct & \\
\hline 13 & CCAGACCAATGGCTGCCAGGGCTTTAGTAAATTTGTGCATgagctcgaattggggatctt & \multirow{2}{*}{$\begin{array}{l}\text { Amplification of } F R T \text {-sacB-tet-FRT } \\
\text { (ay) cassette }\end{array}$} \\
\hline 14 & GTCCGAAATGTGATCGTGGAGTCAATTCTGACGATGATgaattagcttcaaaagcgctct & \\
\hline 15 & GGGGGGAATATTAGGAGGTCGAGatgtctgaaccacgtttcgt & \multirow[t]{2}{*}{ Amplification of $d n a E$ fragment } \\
\hline 16 & GGGGGGAATATTGCATGCttagtcaaactccagttccacc & \\
\hline 17 & CTTTGGGCCTGTCAAACGCGCACTGGGACTTGC GTCCTGgagctcgaattggggatctt & \multirow{2}{*}{$\begin{array}{l}\text { Amplification of } F R T \text {-sacB-dnaE-tet- } \\
F R T(F S D T F) \text { cassette }\end{array}$} \\
\hline 18 & TCAAAATCAAGGAAATTCAGACTCATAGTATTCCTGTAgaattagcttcaaaagcgctct & \\
\hline 19 & actgggacttgcgtcctga & $\begin{array}{l}\text { Detection primer of chromosomal } \\
d n a E\end{array}$ \\
\hline 26 & cattcctttgtggagaacac & $\begin{array}{l}\text { Detection primer of chromosomal } \\
\text { yaeT }\end{array}$ \\
\hline 27 & GGGGGGAATATTAGGAGGCTCGAGttgactactaacactcatactctgc & \multirow[t]{2}{*}{ Amplification of $f a b Z$ fragment } \\
\hline 28 & GGGGGGAATATTGCATGCtcaggcctcccggctacga & \\
\hline 29 & TTATTATTGTCGTTTCTTATATTTTGACAGGAAGAGTATCgagctcgaattggggatctt & \multirow{2}{*}{$\begin{array}{l}\text { Amplification of } F R T \text {-sacB-fabZ-tet- } \\
F R T(F S F T F) \text { cassette }\end{array}$} \\
\hline 30 & TGGCGGTTGGATGCACAAAGGCGGATTTATCAATCACGTAgaattagcttcaaaagcgctct & \\
\hline 31 & cttatattttgacaggaagagtatc & \multirow{2}{*}{$\begin{array}{l}\text { Detection primer of chromosomal } \\
\text { fabZ }\end{array}$} \\
\hline 32 & gcggatttatcaatcacgta & \\
\hline 33 & GGGGGGTCGAGatggcgatgaaaaagttgctca & \multirow{2}{*}{$\begin{array}{l}\text { Amplification of yaeT-hlpA-IpxD frag- } \\
\text { ment }\end{array}$} \\
\hline 34 & GGGGGGGCATGCttagtcttgttgattaaccttgcg & \\
\hline 35 & GGGGGGCTCGAGatgccttcaattcgactggc & \multirow[t]{2}{*}{ Amplification of $I p x D$-fabZ fragment } \\
\hline 36 & GGGGGGGCATGCtcaggcctcccggctacga & \\
\hline 37 & GGGGGGCTCGAGttgactactaacactcatactctgc & \multirow[t]{2}{*}{ Amplification of $f a b Z-I p x A B$ fragment } \\
\hline 38 & GGGGGGGCATGCtcattgtgctaactccagaac & \\
\hline 39 & GGGGGGCTCGAGatgatcgaatttgtttatccgc & \multirow[t]{2}{*}{ Amplification of $r n h B$-dnaE fragment } \\
\hline 40 & GGGGGGGCATGCttagtcaaactccagttccacc & \\
\hline 41 & ccgcttgacgctgcgtaag & \multirow[t]{2}{*}{ Detection primer of plasmid DNA } \\
\hline 42 & ctcatccgccaaaacagcc & \\
\hline
\end{tabular}


Table 3. System Excision Efficiency under Different Conditions

\begin{tabular}{|lccc|}
\hline Conditions & DY2 with pPST & $\begin{array}{c}\text { DYAY with } \\
\text { pBFLPE }\end{array}$ & DYSB \\
\hline $0.05 \%$ arabinose/glycerol & $41 \% / 0 \%$ & $46 \% / 0 \%$ & $37 \% / 0 \%$ \\
$0.1 \%$ arabinose/glycerol & $82 \% / 0 \%$ & $88 \% / 0 \%$ & $79 \% / 0 \%$ \\
$0.2 \%$ arabinose/glycerol & $98 \% / 0 \%$ & $100 \% / 0 \%$ & $94 \% / 0 \%$ \\
$0.3 \%$ arabinose/glycerol & $97 \% / 0 \%$ & $100 \% / 0 \%$ & $92 \% / 0 \%$ \\
$0.4 \%$ arabinose/glycerol & $97 \% / 0 \%$ & $100 \% / 0 \%$ & $90 \% / 0 \%$ \\
$0.5 \%$ arabinose/glycerol & $98 \% / 0 \%$ & $99 \% / 0 \%$ & $93 \% / 0 \%$ \\
One hundred colonies were tested in each experiment and the percentages indicate the number of Suc'/ \\
\hline
\end{tabular}

Table 4. Deletion-complementation Efficiency of yaeT, fabZ, and dnaE Genes

\begin{tabular}{|lccc|}
\hline Plasmids & Transformants & Colony Number & Frequency \\
\hline (A) FSYTF & & & \\
\hline pBFLPE & $(-)$ & 0 & $/$ \\
pBFLPE1 & $(+)$ & 354 & $20 / 20(100 \%)$ \\
pBFLPE2 & $(+)$ & 339 & $20 / 20(100 \%)$ \\
pBFLPE3 & $(-)$ & 0 & $/$ \\
\hline (B) FSFTF & & & $/$ \\
\hline pBFLPE & $(-)$ & 0 & $20 / 20(100 \%)$ \\
pBFLPE2 & $(-)$ & 0 & $20 / 20(100 \%)$ \\
pBFLPE3 & $(+)$ & 398 & $20 / 20(100 \%)$ \\
pBFLPE4 & $(+)$ & 392 & $/$ \\
pBFLPE5 & $(+)$ & 383 & \\
\hline (C) FSDTF & & & \\
\hline pBFLPE & $(-)$ & 0 & $20 / 20(100 \%)$ \\
pBFLPE5 & $(-)$ & 0 & $20 / 20(100 \%)$ \\
pBFLPE6 & $(+)$ & 417 & \\
pBFLPE7 & $(+)$ & 403 & \\
Transformants were selected for Ampr, Sucr, and Tets. $(-)$, no colonies, $(+)$, positive colonies. The next \\
column shows the positive colony number with 100 ng plasmid DNA transformed. In general, 20 colonies \\
were selected in each experiment for PCR and the fractions indicate the number of colonies in which the \\
chromosomal target genes were deleted/20, followed by the calculated frequency in \%.
\end{tabular}

parallel on plates containing sucrose and tetracycline, respectively.

\section{Construction of yaeT, fabZ, or dnaE In-frame Deletion Strains}

FSYTF was transformed with pBFLPE, pBFLPE1, pBFLPE2, and pBFLPE3, respectively. A single colony was picked and cultured to $\mathrm{OD}_{600}=0.3$; the cells were then harvested, spread on M63 plates containing ampicillin and $0.2 \%$ arabinose, and incubated overnight. Control experiments were performed with $0.2 \%$ glycerol. Deletion efficiency of the chromosomal yae $T$ gene and compensatory frequency were detected by PCR. FSFTF was transformed with pBFLPE, pBFLPE2, pBFLPE3, pBFLPE4, and pBFLPE5, while FSDTF was transformed with
pBFLPE, pBFLPE5, pBFLPE6, and pBFLPE7 separately. These constructs were induced and detected as described above.

\section{RESULTS AND DISCUSSION}

In this work, a conditional knockoutrescue system was established to construct an in-frame deletion strain of certain essential genes (Figure 1A). Two FRT sites and positive/negative marker genes were integrated into the $E$. coli DNA, flanking the essential target genes. A plasmid containing arabinose-induced FLP and a constitutively expressed sample gene was transformed into the modified strain. After arabinose induction, cells could survive only when target protein activity was

\section{for all common} dispenser systems

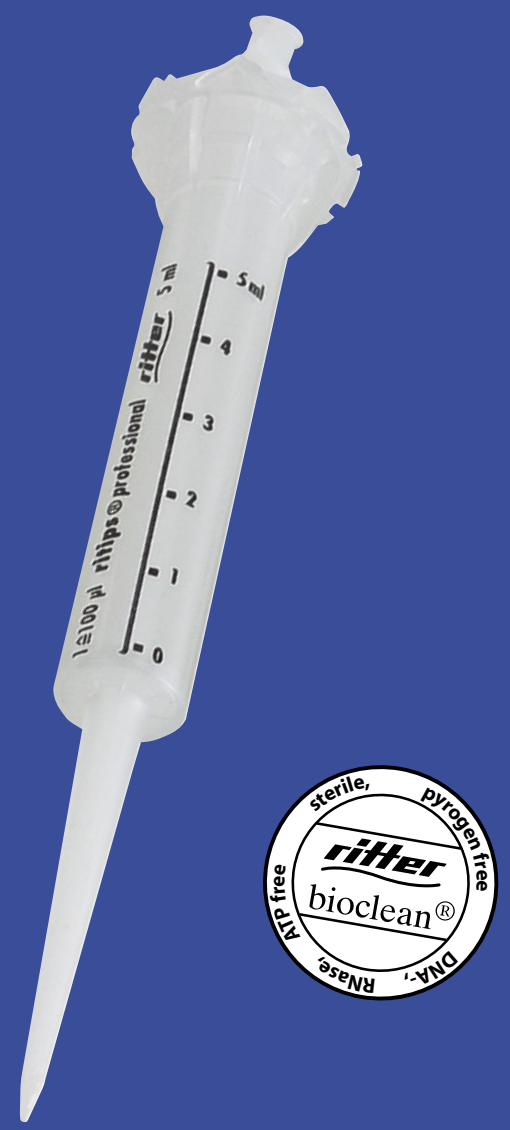

\section{economize by using}

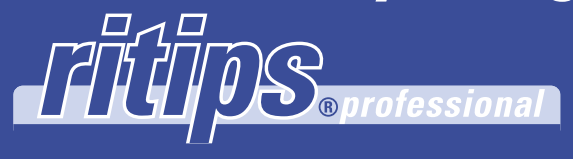

See you at

Pittcon 2008, booth no. 2704

Ritter GmbH

Innovations from Germany

Telephone: +49-(0) 82 32-5003 45 Email: laborbedarf@ritter-online.de

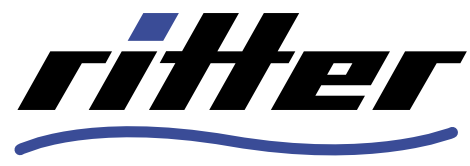

innovations in plastics

www.ritter-medicalcare.de 
provided from a plasmid in trans.

To make this system efficient and suitable for widespread use, the deletion efficiency and the stability of gene expression were considered. We used positive and negative marker genes, tet and $s a c B$, to detect the excision efficiency of FLP recombinase under different conditions; DY2 with pPST, DYAY with pBFLPE, and DYSB were all used to study position effects. After induction with arabinose of different concentrations, the Sucr/Tet ${ }^{\mathrm{s}}$ colonies were numbered to calculate excision efficiency. Results showed that no excision was detected with glycerol. Excision efficiency increased gradually with increasing arabinose concentrations and reached a maximum at $0.2 \%$. Among the three strains, DYAY with pBFLPE exhibited the highest excision efficiency of nearly $100 \%$ (Table 3 ). This is consistent with previous work stating that the excision reaction rate exhibited strong dependence on FLP concentration when FLP was present in excess relative to available recombination sites (26). These experiments were performed in M63 medium as we found that the excision efficiency in M63 medium was higher than that in LB medium (data not shown), perhaps due to the more tightly regulated PBAD expression in $\mathrm{LB}$ medium than in minimal medium (22). All these results indicate that arabinose-controlled FLP is tightly regulated and its excision efficiency is sufficiently high for the study of essential genes.

We further selected three essential genes, yaeT, fabZ, and dnaE, as targets (Figure 1B), to analyze the null mutant construction feasibility of this system. Three strains, FSYTF, FSFTF, and FSDTF, were obtained by flanking the target with both FRT sites and marker genes without changing the promoter(s).

To construct a null mutant of the yaeT gene, several plasmids were transformed into FSYTF. After arabinose induction, positive colonies (Sucr/ Tet $\left.^{s},+\right)$ were only observed in strains containing a second-copy yaeT gene, but no colonies were found in other transformants under any tested conditions (Sucs/Tetr, -). Additionally, PCR results showed that the chromosomal yae $T$ gene was absent and cells could only grow when a copy was provided in trans. Other genes failed to complement this deletion (Table 4A). When fabZ and $d n a E$ genes were used as targets, similar results were found (Table 4, $\mathrm{B}$ and $\mathrm{C}$ ). As expected, no positive transformants were observed in any strains when glycerol was used as an inducer (data not shown). These results indicated that this system could be used for the deletion of essential genes with high efficiency and accuracy. Note that the number of positive transformants (+) could vary from a few to several thousand depending on the quality and amount of plasmid DNA used, the quality of the electrocompetent strains, as well as the electroporation chambers used. But no transformants could be observed in cases indicated by (-).

When we study an operon containing essential genes, one notable question is the polar effects of essential gene deletion on the neighboring genes. To detect whether polar effects exist, we transformed each of the three strains not only with a plasmid harboring the appropriate target essential gene, but also with a plasmid carrying the target and the upstream or downstream gene(s). (Table 1). No differences on cell viability were found (Table 4), which demonstrates that the deletion of the three essential genes (yaeT, fabZ, or $d n a E$ ) did not exert significant polarity effects on neighboring genes.

In summary, this conditional knockout-rescue system completely removed the essential targets from the $E$. coli genome without affecting the function of other genes in the same operon. True null mutants can both eliminate leaky expression and be used in functional studies of genes expressed at low levels. Additionally, the interactions of remaining protein fragments with complementing proteins or other proteins are not possible. Moreover, the target genes expressed under their own promoters before deletion can avoid improper gene expression and undetectable compensatory mutations. Therefore, this system provides a new way for the functional study of essential genes in a complex operon and for screening homologs (e.g., DNA library screening) in other organisms.

\section{ACKNOWLEDGMENTS}

This work was partially supported by the National Basic Research Program of China (grant no. 2005CB724301).

\section{COMPETING INTERESTS STATEMENT}

The authors declare no competing interests.

\section{REFERENCES}

1. Mushegian, A. 1999. The minimal genome concept. Curr. Opin. Genet. Dev. 9:709-714.

2. Armstrong, K.A. and R.K. Herman. 1976. Method for isolation of Escherichia coli $\mathrm{K}$ 12 mutants deficient in essential genes. J. Bacteriol. 126:38-47.

3. Dohmen, R.J., P. Wu, and A. Varshavsky. 1994. Heat-inducible degron: a method for constructing temperature-sensitive mutants. Science 263:1273-1276.

4. Zeidler, M.P., C. Tan, Y. Bellaiche, S. Cherry, S. Hader, U. Gayko, and N. Perrimon. 2004. Temperature-sensitive control of protein activity by conditionally splicing inteins. Nat. Biotechnol. 22:871-876.

5. Guzman, L.M., D. Belin, M.J. Carson, and J. Beckwith. 1995. Tight regulation, modulation, and high-level expression by vectors containing the arabinose PBAD promoter. J. Bacteriol. 177:4121-4130.

6. Mnaimneh, S., A.P. Davierwala, J. Haynes, J. Moffat, W.T. Peng, W. Zhang, X. Yang, J. Pootoolal, et al. 2004. Exploration of essential gene functions via titratable promoter alleles. Cell 118:31-44.

7. Ji, Y., B. Zhang, S.F. Van, P. Warren, G. Woodnutt, M.K. Burnham, and $M$. Rosenberg. 2001. Identification of critical staphylococcal genes using conditional phenotypes generated by antisense RNA. Science 293:2266-2269.

8. Herring, C.D. and F.R. Blattner. 2004 Conditional lethal amber mutations in essential Escherichia coli genes. J. Bacteriol. 186:2673-2681.

9. Schmid, M.B., N. Kapur, D.R. Isaacson, P. Lindroos, and C. Sharpe. 1989. Genetic analysis of temperature-sensitive lethal mutants of Salmonella typhimurium. Genetics 123:625-633.

10. Mohan S., T.M. Kelly, S.S. Eveland, C.R. Raetz., and M.S. Anderson. 1994. An Escherichia coli gene (FabZ) encoding (3R)hydroxymyristoyl acyl carrier protein dehydrase. Relation to $f a b A$ and suppression of mutations in lipid A biosynthesis. J. Biol. Chem. 269:32896-32903.

11. Liu W., C. Luo, C. Han, S. Peng, Y. Yang, J. Yue, X. Shen, and H. Jiang. 2005. A new beta-hydroxyacyl-acyl carrier protein dehydratase (FabZ) from Helicobacter pylori: molecular cloning, enzymatic characterization, 
and structural modeling. Biochem. Biophys. Res. Commun. 333:1078-1086.

12. Gentle, I., K. Gabriel, P. Beech, R. Waller, and T. Lithgow. 2004. The Omp85 family of proteins is essential for outer membrane biogenesis in mitochondria and bacteria. J. Cell Biol. 164:19-24.

13. Doerrler, W.T. and C.R. Raetz. 2005. Loss of outer membrane proteins without inhibition of lipid export in an Escherichia coli YaeT mutant. J. Biol. Chem. 280:2767927687.

14. Werner, J. and R. Misra. 2005. YaeT (Omp85) affects the assembly of lipid-dependent and lipid-independent outer membrane proteins of Escherichia coli. Mol. Microbiol. 57:1450-1459.

15. Wu, T., J. Malinverni, N. Ruiz, S. Kim, T.J. Silhavy, and D. Kahne. 2005. Identification of a multicomponent complex required for outer membrane biogenesis in Escherichia coli. Cell 121:235-245.

16. Sklar J.G., T. Wu, L.S. Gronenberg, J.C. Malinverni, D. Kahne, and T.J. Silhavy. 2007. Lipoprotein SmpA is a component of the YaeT complex that assembles outer membrane proteins in Escherichia coli. Proc. Natl. Acad. Sci. USA. 104:6400-6405.

17. Robert, V., E.B. Volokhina, F. Senf, M.P. Bos, P. Van Gelder, and J. Tommassen. 2006. Assembly factor Omp85 recognizes its outer membrane protein substrates by a species-specific C-terminal motif. PLoS Biol. 4: e377.

18. Voulhoux, R., M.P. Bos, J.M. Geurtsen Mols, and J. Tommassen. 2003. Role of a highly conserved bacterial protein in outer membrane protein assembly. Science 299:262-265.

19. Lo T., E. van Der Schalie, T. Werner, Y.V. Brun, and N. Din. 2004. A temperature-sensitive mutation in the $d n a E$ gene of Caulobacter crescentus that prevents initiation of DNA replication but not ongoing elongation of DNA. J. Bacteriol. 186:1205-1212.

20. Dohrmann, P.R. and C. S. McHenry. 2005. A bipartite polymerase-processivity factor interaction: only the internal beta binding site of the alpha subunit is required for processive replication by the DNA polymerase III holoenzyme. J. Mol. Biol. 350:228-239.

21. Miller, J.H. 1972. Experiments in Molecular Genetics. CSH Laboratory Press, Cold Spring Harbor, NY.

22. Guzman, L.M. 1992. FstL, an essential cytoplasmic membrane protein involved in cell division in Escherichia coli. J. Bacteriol. 174:7716-7728.

23. Woodcock, D.M., P.J. Crowther, J. Doherty, S. Jefferson, E. DeCruz, M. Noyer-Weidner, S.S. Smith., M.Z. Michael, and M.W. Graham. 1989. Quantitative evaluation of Escherichia coli host strains for tolerance to cytosine methylation in plasmid and phage recombinants. Nucleic Acids Res. 17:3469-3478.

24. Hoang, T.T., R.R. Karkhoff-Schweizer, A.J. Kutchma, and H.P. Schweizer. 1998. A broad-host-range Flp- $F R T$ recombination system for site-specific excision of chromosomally located DNA sequences: application for isolation of unmarked Pseudomonas aeruginosa mutants. Gene 212:77-86.

25. Yu, D., H.M. Ellis, E.C. Lee, N.A. Jenkins, N.G. Copeland, and D.L. Court. 2000. An efficient recombination system for chromosome engineering in Escherichia coli. Proc. Natl. Acad. Sci. USA 97:5978-5983.

26. Gates, C.A. and M.M. Cox. 1988. FLP recombinase is an enzyme. Proc. Natl. Acad. Sci. USA 85:4628-4632.

Received 6 December 2006; accepted 30 October 2007.

Address correspondence to Rubing Liang, College of Life Science \& Technology, Shanghai Jiaotong University, 800 DongChuan Road, Shanghai 200240, China. e-mail: jianhualiudl@sjtu.edu.cn

To purchase reprints of this article, contact: Reprints@BioTechniques.com 